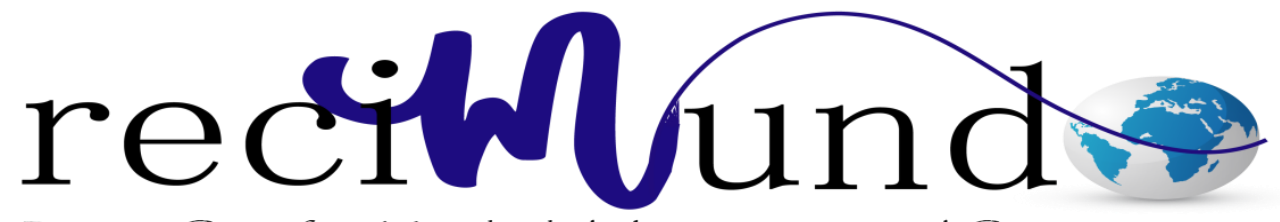

Revista Cientifica Mundo de la Investigación y el Conocimiento

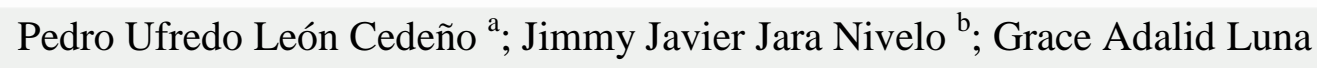
Yerovi $^{\mathrm{c}}$

Elaboración de flujo efectivo para lograr financiamiento a largo plazo de una empresa de fertilizante

Revista Científica Mundo de la Investigación y el Conocimiento. Vol. 2 núm., especial, mayo, ISSN: 2588-073X, 2018, pp. 136-156

DOI: 10.26820/recimundo/2.esp.2018.136-156

Editorial Saberes del Conocimiento

Recibido: 20/12/2017

Aceptado: 26/02/2018
a. Universidad de Guayaquil.
b. Universidad de Guayaquil.
c. Universidad de Guayaquil. 


\section{Elaboración de flujo efectivo para lograr financiamiento a largo plazo de una empresa de fertilizante \\ Vol. 2, núm. Esp., (2018) \\ Pedro Ufredo León Cedeño; Jimmy Javier Jara Nivelo; Grace Adalid Luna Yerovi}

\section{RESUMEN}

Introducción: A pesar del avance pujante de los últimos años, y al igual que la mayoría de los países latinoamericanos, su capital sigue dependiendo de las exportaciones de materias primas y el petróleo es la principal fuente de riqueza del país. Ecuador, como todos los países de América Latina, se independizó porque quería tener poder político y libertad para desarrollar sus actividades económicas; los criollos consideraban injusto estar excluidos de la política y de las decisiones financieras. Objetivo: Elaborar Flujos Efectivos para lograr financiamiento a largo plazo de una empresa de fertilizantes. Metodología: La investigación se despliega en parámetros descriptivos, explorativo, explicativo y de campo ya que emplearemos el diseño mixto, el mismo que lo aplicaremos una sola vez en el tiempo, y la herramientas que se utilizará son la entrevista y observación las cuales proporcionarán información relevante y fidedigna con el propósito de entender, verificar, corregir o aplicar el conocimiento. Resultados: Con las entrevistas realizadas al representante legal, gerente de la empresa y el contador, se puede determinar que la empresa posee un abaja liquidez debido principalmente a que no genera muchos ingresos y en segunda estancia se obtiene que la empresa no realiza gestión del movimiento de capital que realiza. Conclusiones: Se pudo constatar los problemas financieros de la empresa, el implementar una herramienta que ayude en la gestión de efectivo, de esta manera la empresa obtenga una mejor liquidez al finalizar el periodo contable.

Palabras claves: Flujo efectivo; empresa de fertilizantes; periodo contable. 


\title{
Elaboración de flujo efectivo para lograr financiamiento a largo plazo de una empresa de fertilizante
}

Vol. 2, núm. Esp., (2018)

Pedro Ufredo León Cedeño; Jimmy Javier Jara Nivelo; Grace Adalid Luna Yerovi

\begin{abstract}
Introduction: Despite the booming progress of recent years, and like most Latin American countries, its capital continues to depend on exports of raw materials and oil is the main source of wealth for the country. Ecuador, like all the countries of Latin America, became independent because it wanted to have political power and freedom to develop its economic activities; the criollos considered it unjust to be excluded from politics and financial decisions. Objective: Develop Cash Flows to obtain long-term financing from a fertilizer company. Methodology: The research is deployed in descriptive, exploratory, explanatory and field parameters since we will use the mixed design, the same one that we will apply only once in time, and the tools that will be used are the interview and observation which will provide information relevant and reliable in order to understand, verify, correct or apply knowledge. Results: With the interviews made to the legal representative, the company manager and the accountant, it can be determined that the company has a low liquidity mainly due to the fact that it does not generate a lot of income and in the second stay it is obtained that the company does not manage the movement of capital that it realizes. Conclusions: It was possible to verify the financial problems of the company, to implement a tool that helps in cash management, in this way the company obtains a better liquidity at the end of the accounting period.
\end{abstract}

Keywords: Cash flow; fertilizer company; accounting period. 


\section{Elaboración de flujo efectivo para lograr financiamiento a largo plazo de una}

empresa de fertilizante

Vol. 2, núm. Esp., (2018)

Pedro Ufredo León Cedeño; Jimmy Javier Jara Nivelo; Grace Adalid Luna Yerovi

\section{Introducción.}

A pesar del avance pujante de los últimos años, y al igual que la mayoría de los países latinoamericanos, su capital sigue dependiendo de las exportaciones de materias primas y el petróleo es la principal fuente de riqueza del país. Ecuador, como todos los países de América Latina, se independizó porque quería tener poder político y libertad para desarrollar sus actividades económicas; los criollos consideraban injusto estar excluidos de la política y de las decisiones financieras. (Vargas Soto, 2007) (Garrido \& Granados, 2004)

El periodo 1907-1918, crecen las relaciones comerciales con Francia, Estados Unidos, Gran Bretaña y Alemania. Las exportaciones con estos países ocupan entre el 70 y $80 \%$ del total, siendo básicamente productos agrícolas: cacao $64,1 \%$ y café $5,4 \%$, de este modo la economía creció, pero se volvió más dependiente del mercado internacional. (Finnerty, 1998) (Durán, 2011) (Páez, 2007)

En el Ecuador para poder realizar las actividades productivas las compañías deben buscar otras alternativas de financiamiento entre las cuales se puede mencionar la inversión extranjera ya que la compañía carece de financiamiento.

En la Ley de Mercado de Valores del Ecuador, las empresas pueden realizar operaciones para obtener financiamiento para actividades de capital de trabajo, compras de activos fijos y restructuración de pasivos.

El mercado de valores es importante para el desarrollo de la economía de un país. En el Ecuador no se ha podido alcanzar un nivel adecuado de financiamiento, debido a la escasa oferta 


\section{Elaboración de flujo efectivo para lograr financiamiento a largo plazo de una empresa de fertilizante}

Vol. 2, núm. Esp., (2018)

Pedro Ufredo León Cedeño; Jimmy Javier Jara Nivelo; Grace Adalid Luna Yerovi

de títulos, valores, por lo que se hace necesaria la estructuración de financiamientos a largo plazo

para realizar el proceso de titularización que faciliten el crecimiento del mercado y el ingreso de nuevos participantes en el mismo.

El Apalancamiento Financiero es como se denomina en finanzas al grado en que una empresa depende de su deuda, es decir, cuánto llega a recurrir una empresa durante su tiempo de vida a los préstamos, de instituciones financieras que ayudan en esta gestión de capitalizar a la empresa. El propósito puede ser reestructurar su capital, ampliar una línea de producción, o simplemente financiar alguna actividad relacionada de forma directa con el giro de la empresa.

El financiamiento se utiliza principalmente para reestructurar el capital de la empresa y, ante esto, debe tenerse en cuenta que cuanto más financiamiento de la deuda utiliza una empresa en la estructura de capital, mayor es el uso del apalancamiento financiero.

La empresa de fertilizante que es ejemplo de nuestro estudio, no está exenta de estas necesidades en el flujo de caja, por lo que es necesario un plan para lograr un mejor apalancamiento financiero en emisiones de bonos, acciones, hipotecas.

El presente estudio tuvo como objetivo elaborar Flujos Efectivos para lograr financiamiento a largo plazo de una empresa de fertilizantes. 


\section{Elaboración de flujo efectivo para lograr financiamiento a largo plazo de una \\ empresa de fertilizante}

Vol. 2, núm. Esp., (2018)

Pedro Ufredo León Cedeño; Jimmy Javier Jara Nivelo; Grace Adalid Luna Yerovi

\section{Metodología.}

Tipo y diseño de investigación

La investigación se despliega en parámetros descriptivos, explorativo, explicativo y de campo ya que emplearemos el diseño mixto, el mismo que lo aplicaremos una sola vez en el tiempo, y la herramientas que se utilizará son la entrevista y observación las cuales proporcionarán información relevante y fidedigna con el propósito de entender, verificar, corregir o aplicar el conocimiento.

Para equitativamente obtener beneficios de las partes, la investigación descriptiva se desarrolla en la aplicación de herramientas con el propósito de elaborar el diagnóstico real de necesidad, dar respuestas a las preguntas y analizar científica y técnicamente la propuesta mencionada.

Investigación descriptiva: Mediante este tipo de investigación, que utiliza el método de análisis, se logra caracterizar un objeto de estudio o una situación concreta, señalar sus características y propiedades. Combinada con ciertos criterios de clasificación que sirven para ordenar, agrupar o sistematizar los objetos involucrados en el trabajo indagatorio.

Investigación explicativa: Mediante esta ideal investigación, que requiere la combinación de los métodos analítico y sintético, en conjugación con el deductivo y el inductivo, se trata de responder o dar cuenta del porqué del objeto que se investiga.

Investigación campo: Sirve de apoyo en informaciones que provienen de entrevistas, cuestionarios, encuestas y observaciones. En esta se obtiene la información directamente 


\section{Elaboración de flujo efectivo para lograr financiamiento a largo plazo de una empresa de fertilizante}

Vol. 2, núm. Esp., (2018)

Pedro Ufredo León Cedeño; Jimmy Javier Jara Nivelo; Grace Adalid Luna Yerovi

en la realidad en que se encuentra, por lo tanto, implica observación directa por parte del investigador.

Cualitativa: Es un método de investigación usado principalmente en las ciencias sociales que se basa en cortes metodológicos basados en principios teóricos tales como la fenomenología, la hermenéutica, la interacción social empleando métodos de recolección de datos que son no cuantitativos, con el propósito de explorar las relaciones sociales y describir la realidad tal como la experimentan sus correspondientes protagonistas. La investigación cualitativa requiere un profundo entendimiento del comportamiento humano y las razones que lo gobiernan.

\section{Población y muestra.}

\section{Características de la población}

Los sujetos que ya fueron estudiados para el objeto de investigación sobre la problemática planteada son los empleados y los socios de los cuales se les aplicará una herramienta investigativa para obtener información relevante sobre la elaboración de un flujo efectivo para lograr el financiamiento a largo plazo de una empresa de fertilizante. Tomando en consideración que actualmente cuenta con 40 empleados y 7 directivos que se encuentra laborando en la empresa en mención. 


\section{Elaboración de flujo efectivo para lograr financiamiento a largo plazo de una}

empresa de fertilizante

Vol. 2, núm. Esp., (2018)

Pedro Ufredo León Cedeño; Jimmy Javier Jara Nivelo; Grace Adalid Luna Yerovi Delimitación de la población

Las técnicas de la encuestas se realzará a directivos de la empresa, de la misma manera se realiza un entrevista al contador, debido a que se considera que estos individuos tienen un amplio conocimiento acerca del tema a tratar.

Proceso de selección

Uno de los tipos de muestras es NO probabilístico se tomará el proceso de selección intencional a los individuos que se les aplicara la técnica de la investigación.

Los métodos y las técnicas

Los métodos e instrumentos que aplicaremos en el estudio de la problemática planteada son los siguientes.

\section{Métodos teóricos}

Método hipotético deductivo: Este método tiene un alto grado de significación de aquellas ciencias muy sistematizadas y cuyo objeto de estudio es relativamente sencillo y posible de abstraer.

Análisis - Síntesis: El análisis y la síntesis son dos procesos cognoscitivos que cumplen funciones muy importantes en la investigación científica.

El análisis: Es una operación intelectual que posibilita descomponer mentalmente un todo complejo en sus partes y cualidades. 


\section{Elaboración de flujo efectivo para lograr financiamiento a largo plazo de una empresa de fertilizante}

Vol. 2, núm. Esp., (2018)

Pedro Ufredo León Cedeño; Jimmy Javier Jara Nivelo; Grace Adalid Luna Yerovi

La síntesis: La síntesis establece mentalmente la unión entre las partes previamente analizadas y posibilita descubrir las relaciones esenciales y características generales entre ellas

Desintegración de un todo en sus partes o elementos para observar y establecer causas y efectos de uno o varios fenómenos del ambiente que se establece en la entidad.

Método estadístico: Por medio de este método vamos a darle tratamiento a la información que se obtendrá a través de la encuesta.

\section{Técnica e instrumentos}

Las técnicas utilizadas para la recolección de información se las realizó mediante las siguientes maneras.

La entrevista.- al igual que la encuesta es un instrumento investigativo que sirve para obtener información de un tema específico desde el punto de vista de un profesional en el área.

La observación.- Es una técnica que consiste en observar atentamente el fenómeno, hecho o caso, tomar información y registrarla para su posterior análisis. La observación es un elemento fundamental de todo proceso investigativo; en ella se apoya el investigador para obtener el mayor número de datos. 


\section{Elaboración de flujo efectivo para lograr financiamiento a largo plazo de una}

empresa de fertilizante

Vol. 2, núm. Esp., (2018)

Pedro Ufredo León Cedeño; Jimmy Javier Jara Nivelo; Grace Adalid Luna Yerovi El tratamiento estadístico de la información

El procesamiento estadístico de la información se la realizará a través de la recolección de datos obtenidos de la entrevista, los mismos que serán analizadas e interpretados con un informe de recomendaciones y conclusiones.

Análisis e interpretación de los datos obtenidos en la investigación

1. ¿Está de acuerdo con la situación financiera actual de la empresa de fertilizante?

2. ¿Le gustaría que la empresa expanda su negocio?

3. ¿Qué estrategia debe tomar la empresa para su crecimiento?

4. ¿A su criterio con la utilización de F/E podemos determinar si el financiamiento con acciones comunes es posible la compra de activos fijos para el crecimiento de la empresa fertilizante?

5. ¿Qué tasa de interés estaría dispuesto a pagar la empresa por la emisión de Acciones comunes?

6. ¿A qué tasa de descuento negociarían las acciones comunes en el mercado?

7. ¿Conoce usted alguna empresa haya utilizado este tipo de financiamiento a largo plazo?

8. ¿Que necesitara la empresa para operar bajo este sistema de financiamiento?

9. ¿Considera usted que en el mercado de valores las acciones comunes emitidas por la empresa, tenga la acogida por los inversionistas?

10. ¿Está de acuerdo que la implementación de la propuesta beneficiara a la empresa? 
Elaboración de flujo efectivo para lograr financiamiento a largo plazo de una empresa de fertilizante

Vol. 2, núm. Esp., (2018)

Pedro Ufredo León Cedeño; Jimmy Javier Jara Nivelo; Grace Adalid Luna Yerovi

\section{Entrevista aplicada a directivos y contador de la empresa}

\section{ENTREVISTA \# 1}

\section{Tema a tratar:}

ELABORACION DE FLUJO EFECTIVO PARA LOGRAR FINANCIAMIENTO A LARGO PLAZO DE UNA EMPRESA DE FERTILIZANTE

Nombre: Ing. Galo Álava

Cargo: Representante legal de la empresa

1. ¿Está de acuerdo con la situación financiera actual de la empresa de fertilizante?

Actualmente la empresa posee una baja liquidez debido a que no se tiene la gestión de efectivo ni algún tipo de financiamiento que aumente los niveles de ingresos.

2. ¿Le gustaría que la empresa expanda su negocio?

Como todo negocio debe crecer, pero para esto se debe tener el capital necesario para la adquisición de nuevos bienes.

3. ¿Qué estrategia debe tomar la empresa para su crecimiento?

Principalmente se debe capitalizar, es decir generar ingresos ya sean por entidades bancarias, captación de nuevos socios o incrementando los niveles de ventas en años posteriores, mientras más capital posea la empresa se puede expandir hacia nuevos mercados.

4. ¿A su criterio con la utilización de F/E podemos determinar si el financiamiento con acciones comunes es posible la compra de activos fijos para el crecimiento de la empresa fertilizante? 


\section{Elaboración de flujo efectivo para lograr financiamiento a largo plazo de una}

empresa de fertilizante

Vol. 2, núm. Esp., (2018)

Pedro Ufredo León Cedeño; Jimmy Javier Jara Nivelo; Grace Adalid Luna Yerovi

Con la venta de acciones y que la empresa se encuentre con una liquidez positiva, es posible la compra de nuevos bienes para el crecimiento de la empresa.

5. ¿Qué tasa de interés estaría dispuesto a pagar la empresa por la emisión de acciones comunes?

La tasa de interés mínima, o dependiendo de la relación que se pueda generar con nuevos socios.

\section{6. ¿A qué tasa de descuento negociarían las acciones comunes en el mercado?}

Para que las acciones de la empresa se puedan negociar en el mercado, se debe contar con una liquidez y niveles de financiamientos que mantenga en estabilidad en la organización. El valor de cada acción y descuento depende del nivel de financiamiento de la empresa, debido a que la empresa se encuentra con una liquidez baja es posible que esta se negocie por debajo de la tasa mínima.

7. ¿Conoce usted alguna empresa haya utilizado este tipo de financiamiento a largo plazo?

Para muchas empresas el uso de métodos para obtener financiamiento a largo plazo depende de la relación que tenga la empresa con los socios, existen entidades que deben cancelar un porcentaje por llevar la imagen de la marca.

8. ¿Que necesitará la empresa para operar bajo este sistema de financiamiento? 


\section{Elaboración de flujo efectivo para lograr financiamiento a largo plazo de una empresa de fertilizante}

Vol. 2, núm. Esp., (2018)

Pedro Ufredo León Cedeño; Jimmy Javier Jara Nivelo; Grace Adalid Luna Yerovi

Mejorar la situación de liquidez, presentar un informe a diferentes individuos los cuales pueden ser los nuevos socios de la empresa, realizar una negociación donde se especifique el aporte que debe cancelar y los beneficios que pueden obtener.

9. ¿Considera usted que en el mercado de valores las acciones comunes emitidas por la empresa, tenga la acogida por los inversionistas?

Con una mejor liquidez y realizar una presentación económica de la empresa la venta de acciones se puede generar de una manera automática, es decir todo individuo que observe los niveles e liquidez toma en consideración la idea de adquirir acciones.

\section{0. ¿Está de acuerdo que la implementación de la propuesta beneficiara a la empresa?}

El principal beneficio que se puede obtener gracias al flujo de caja es mejorar la gestión de capital y especificar los motivos por los cuales se generó un ingreso o un gasto, de esta manera se podrá obtener un mejor control de los ingresos y gastos que realiza la empresa. 
Elaboración de flujo efectivo para lograr financiamiento a largo plazo de una empresa de fertilizante

Vol. 2, núm. Esp., (2018)

Pedro Ufredo León Cedeño; Jimmy Javier Jara Nivelo; Grace Adalid Luna Yerovi

\begin{tabular}{|l|l|}
\hline ENTREVISTA \# 2 \\
Tema a tratar: \\
ELABORACION DE FLUJO EFECTIVO PARA LOGRAR \\
FINANCIAMIENTO A LARGO PLAZO DE UNA EMPRESA DE \\
FERTILIZANTE \\
\hline Nombre: Ing. Jaime Ulloa \\
Cargo: Gerente general de la empresa
\end{tabular}

1. ¿Está de acuerdo con la situación financiera actual de la empresa de fertilizante?

Debido al mal manejo de los recursos y de capital, la empresa se encuentra en una situación que puede llevarla al cierre permanente, en los últimos estados financieros se puede observar el nivel de financiamiento, liquidez y solvencia de la empresa son bajos en comparación con estados financieros anteriores.

2. ¿Le gustaría que la empresa expanda su negocio?

Claro que sí, expansión de negocio es sinónimo de abarcar mercado y al hacer eso la empresa puede adquirir nuevos clientes y nuevos ingresos.

3. ¿Qué estrategia debe tomar la empresa para su crecimiento?

Fomentar la alianza con empresas o individuos que les gustaría ser partícipe de la actividad comercial de la empresa.

4. ¿A su criterio con la utilización de F/E podemos determinar si el financiamiento con acciones comunes es posible la compra de activos fijos para el crecimiento de la empresa fertilizante? 


\section{Elaboración de flujo efectivo para lograr financiamiento a largo plazo de una empresa de fertilizante}

Vol. 2, núm. Esp., (2018)

Pedro Ufredo León Cedeño; Jimmy Javier Jara Nivelo; Grace Adalid Luna Yerovi

Con la obtención de nuevos ingresos, siempre es recomendable adquirir nuevos bienes que ayuden a incrementar los niveles de producción. Las ventas de acciones de la empresa pueden generar ingresos suficientes para cumplir este propósito.

\section{5. ¿Qué tasa de interés estaría dispuesto a pagar la empresa por la emisión acciones comunes?}

Considero, la que sea necesaria para mejorar la liquidez y forjando nuevas relaciones comerciales pero siempre y cuando se obtenga resultados positivos y que estos beneficien a la organización.

6. ¿A qué tasa de descuento negociarían las acciones comunes en el mercado?

Para empezar las ventas de acciones, asumo que se puede negociar la mitad de cada valor, es decir vender cada acción a mitad de precio, si el financiamiento es el correcto y se genera suficiente liquidez para la empresa, se puede recuperar el capital a largo plazo.
7. ¿Conoce usted alguna empresa haya utilizado este tipo de financiamiento a largo plazo?

Varias empresas obtienen diferentes ingresos y se debe a las relaciones comerciales o alianzas estratégicas entre demás empresas o personas que desean entrar al campo de negocios.

8. ¿Que necesitara la empresa para operar bajo este sistema de financiamiento? 


\section{Elaboración de flujo efectivo para lograr financiamiento a largo plazo de una}

empresa de fertilizante

Vol. 2, núm. Esp., (2018)

Pedro Ufredo León Cedeño; Jimmy Javier Jara Nivelo; Grace Adalid Luna Yerovi El método más factible es generar nuevas relaciones comerciales consocios que puedan expandir el negocio con el fin de obtener otros ingresos que ayuden a mejorar los niveles de liquidez de la empresa.

9. ¿Considera usted que en el mercado de valores de las acciones comunes emitidas por la empresa, tenga la acogida por los inversionistas?

Cuando una empresa posee una economía sostenible con una visión de evolución, de manera inesperada existirán individuos que querrán obtener beneficios con solo el ingreso de capital para aportar a la empresa.

\section{0. ¿Está de acuerdo que la implementación de la propuesta beneficiará a la empresa?}

En si el flujo de efectivo ayudará a gestionar el capital que maneja la empresa y con el financiamiento de acciones aparte de obtener otro ingreso, facilitará el análisis y la toma de decisiones para adquirir nuevos bienes, disminuir costos o cualquier método necesario para beneficio en razón de liquidez para la empresa. 
Elaboración de flujo efectivo para lograr financiamiento a largo plazo de una empresa de fertilizante

Vol. 2, núm. Esp., (2018)

Pedro Ufredo León Cedeño; Jimmy Javier Jara Nivelo; Grace Adalid Luna Yerovi

\section{ENTREVISTA \# 3}

\section{Tema a tratar:}

ELABORACION DE FLUJO EFECTIVO PARA LOGRAR FINANCIAMIENTO A LARGO PLAZO DE UNA EMPRESA DE FERTILIZANTE

Nombre: CPA. Mario Lindao

Cargo: Contador de la empresa

\section{1. ¿Está de acuerdo con la situación financiera actual de la empresa de fertilizante?}

En el último ejercicio contable la empresa reflejo una baja liquidez donde se pudo determinar que la empresa genera un alto índice de gastos y pocos ingresos.

\section{2. ¿Le gustaría que la empresa expanda su negocio?}

Debería, abarcar nuevos mercados es generar nuevas relaciones comerciales y estas con la adecuada negociación para beneficio mutuo se crean sociedades que puedan expandir el negocio.

\section{3. ¿Qué estrategia debe tomar la empresa para su crecimiento?}

Obtener nuevos bienes e ingresos que ayuden a incrementar los niveles de liquidez de la empresa.

4. ¿A su criterio con la utilización de F/E podemos determinar si el financiamiento con acciones comunes es posible la compra de activos fijos para el crecimiento de la empresa fertilizante?

Es una de las estrategias que pueden ayudar a mejorar la situación de la empresa, como dicho anteriormente, obtener nuevos ingresos se realiza la compra de nuevos activos con los cuales se puede incrementar los niveles de ventas, dando un beneficio para la empresa. 


\section{Elaboración de flujo efectivo para lograr financiamiento a largo plazo de una}

empresa de fertilizante

Vol. 2, núm. Esp., (2018)

Pedro Ufredo León Cedeño; Jimmy Javier Jara Nivelo; Grace Adalid Luna Yerovi

5. ¿Qué tasa de interés estaría dispuesto a pagar la empresa por la emisión de acciones comunes?

Realizar la venta de acciones con tasa de interés, considero que debe ser determinada por la parte administrativa.

6. ¿A qué tasa de descuento negociarían las acciones comunes en el mercado?

Con relación a la pregunta anterior los valores de cada acción deben ser determinados por la parte administrativa.

7. ¿Conoce usted alguna empresa haya utilizado este tipo de financiamiento a largo plazo?

En la empresa que laboraba anteriormente se obtenía ingresos por publicidad los cuales se originaba debido a la relación que se obtenía con demás empresa asociadas para auspiciar los productos y que sean comercializados dentro de las instalaciones.

8. ¿Que necesitara la empresa para operar bajo este sistema de financiamiento?

Que el valor de las acciones se incremente para atraer nuevos socios, pero esto se puede realizar mejorando la liquidez de la empresa.

9. ¿Considera usted que en el mercado de valores de las acciones comunes emitidas por la empresa, tenga la acogida por los inversionistas? 


\section{Elaboración de flujo efectivo para lograr financiamiento a largo plazo de una empresa de fertilizante}

Vol. 2, núm. Esp., (2018)

Pedro Ufredo León Cedeño; Jimmy Javier Jara Nivelo; Grace Adalid Luna Yerovi

Con las ventas de acciones especialmente de una empresa que se encuentra con una buena liquidez, esto se genera de manera rápida.

\section{0. ¿Está de acuerdo que la implementación de la propuesta beneficiara a la empresa?}

Cualquier estrategia que ayude a obtener mayores ingresos ya sea por cuenta de la empresa o por ayuda de relaciones con socios que se puedan generar, la empresa de encontrase con una situación financiera seria, es decir que presente una liquidez positiva.

\section{Análisis de los resultados obtenidos}

Con las entrevistas realizadas al representante legal, gerente de la empresa y el contador, se puede determinar que la empresa posee un abaja liquidez debido principalmente a que no genera muchos ingresos y en segunda estancia se obtiene que la empresa no realiza gestión del movimiento de capital que realiza.

Las tres personas entrevistadas están de acuerdo en que la empresa necesita una fuente de ingreso que ayude a incrementar los niveles de capital que obtiene la empresa. Si bien es cierto que la entidad no posee una liquidez suficiente para que se mantenga vigente en el mercado, esta no genera el interés para atraer nuevos socios para forjar nuevas relaciones para beneficio mutuo.

La herramienta que se desea plantear para la resolución de la problemática encontrada, es un diseño de flujo de caja y obtener un financiamiento por venta de acciones que se pueda generar a largo plazo, el cual tiene como fin proporcionar una herramienta que le ayude a gestionar los movimientos de efectivo y mejorar los ingresos que se realicen para beneficio de la empresa, también se puede asimilar que si la empresa se proyecta un flujo de efectivo positivo 


\section{Elaboración de flujo efectivo para lograr financiamiento a largo plazo de una}

empresa de fertilizante

Vol. 2, núm. Esp., (2018)

Pedro Ufredo León Cedeño; Jimmy Javier Jara Nivelo; Grace Adalid Luna Yerovi que se puede generar de manera oportuna la venta de más acciones para nuevas relaciones comerciales o inclusive préstamos para que de esta manera se obtenga otro ingreso e incrementando los niveles de financiamiento.

\section{Conclusiones.}

Se pudo constatar los problemas financieros de la empresa, el implementar una herramienta que ayude en la gestión de efectivo, de esta manera la empresa obtenga una mejor liquidez al finalizar el periodo contable.

Se determina que la empresa lleva un inadecuado proceso para el control de los gastos y/o ingresos que se realizan las actividades comerciales, debido a que no realiza constataciones de manera física, en reiteradas ocasiones los valores que se registran en los balances son asumidos por parte del personal competente, lo que ocasiona un desconocimiento del manejo de capital que realiza la empresa.

Debido al mal manejo de registros que realiza el personal implicado en esta actividad, se puede asumir que no está totalmente capacitado para el cargo o desconoce todo el procedimiento contable que ayude a reflejar la situación económica, financiera y tributaria de la empresa.

Las herramientas utilizadas como técnicas de investigación, ayudaron a determinar e identificar los problemas que afronta la empresa, se obtuvo como resultado la estructura actual y el proceso contable que maneja el personal competente de la empresa, estas técnicas dieron las pautas para priorizar el problema y determinar una propuesta que ayude a mejorar la situación que afrontan el cual es debido principalmente a la falta de ingresos. 


\section{Elaboración de flujo efectivo para lograr financiamiento a largo plazo de una empresa de fertilizante}

Vol. 2, núm. Esp., (2018)

Pedro Ufredo León Cedeño; Jimmy Javier Jara Nivelo; Grace Adalid Luna Yerovi

\section{Bibliografía.}

Durán, Y. (2011). Administración del Capital de Trabajo: una herramienta financiera para la gerencia de las PyME tradicionales venezolanas. Visión Gerencial, 1(ene), 37-56.

Finnerty, J. (1998). Financiamiento de proyectos. México: Pearson, Prentice Hall.

Garrido, C., \& Granados, L. (2004). Innovación, financiamiento y organización financiera nacional. Problemas del Desarrollo. Revista Latinoamericana de Economía, 35(139), 163-184.

Páez, T. (2007). eoría y práctica de la ética y la responsabilidad social de la PyME venezolana. Revista FACES, 17(5), 165-183.

Vargas Soto, R. (2007). ESTADO DE FLUJO DE EFECTIVO. InterSedes: Revista de las Sedes Regionales, 7(14), 111-136. 